\title{
A New Five Parameter Lifetime Distribution: Properties and Application
}

\author{
Ahmed M. T. Abd El-Bar ${ }^{1}$, Shimaa A. Dessoky ${ }^{2}$ \\ Mathematics Department, Faculty of Science, Tanta University, Tanta, Egypt \\ Mathematics Department, Faculty of Science, Tanta University, Tanta, Egypt
}

\begin{abstract}
This paper deals with a new generalization of the Weibull distribution. This distribution is called exponentiated exponentiated exponential-Weibull (EEE-W) distribution. Various structural properties of the new probabilistic model are considered, such as hazard rate function, moments, moment generating function, quantile function, skewness, kurtosis, Shannon entropy and Rényi entropy. The maximum likelihood estimates of its unknown parameters are obtained. Finally, areal data set is analyzed and it observed that the present distribution can provide a better fit than some other known distributions.
\end{abstract}

\section{Indexing terms/Keywords}

T-X family; Moment generating function; Quantile function; Maximum likelihood estimation.

\section{SUBJECT CLASSIFICATION}

Mathematics Subject Classification: 62E10;62E15; 62F10

\section{INTRODUCTION}

The statistic literature is filed with hundred of continuous univariat distributions; see Johnson et. al., [1, 2]. Recent developments focus on new techniques for building meaningful distributions, including the two piece approach introduced by Hansen [3], the perturbation approach of Azzalini and Capitanio [4] and the generator approach pioneered by Eugene et. al., [5]. Many subsequent articles apply these techniques to introduce a skew in to well-known symmetric distributions such as the Student t; see Aas and Haff [6]. Numerous classical distributions have been extensively used over the bast decades for modeling data in several areas such as engineering and actuarial. However in many applied areas there is a clear need for extended forms of these distributions. Some attempts have been made to define new families of probability distribution. One such example is a abroad family of univariate distributions generated from the Weibull distribution introduced by Gurvich et. al., [7].

The Weibull distribution is a very popular model and it has been extensively used over the past decades for modeling data in reliability engineering and bio-logical studies. It is generally adequate for modeling monotone hazard rates. The hazard rate function of the Weibull distribution can only be increasing, decreasing or constant. For many years, researchers have been developing various extensions forms of the Weibull distribution. For some extended forms of the Weibull distribution (see, for instance, Bebbington et. al., [8], Zhang and Xie [9], Xie et. al., [10], Carrasco et. al., [11], Sarhan and Zaindin [12] and Almalki and Yuan [13]. Also, Xie and Lai [14] proposed a four-parameter additive Weibull (AW) distribution based on combining the failure rates of tow Weibull distributions: one has a decreasing failure rate and the other one has an increasing failure rate.

In the last years, several ways of generating new probability distributions were developed and discussed. Eugene et. al., [5] proposed a general class of distributions based on the logit of a beta random variable by employing two parameters whose role is to introduce Skewness and to vary tail weights. An extension of the beta-generated method was proposed in Cordiro and de Castro [15] by using the Kumaraswamy distribution instead of beta distribution. Following Eugene et al [5], who defined the Beta Normal (BN) distribution. Nadaraga and Kotz [16] introduce the beta Gumbel distribution (BGa). Nadaraga and Gupta [17] defined the Beta Fréchet (BF) distribution. Nadaraga and Kotz [18] proposed the beta exponential (BE) distribution.

Recently, Alzaatreh et. al., [19] developed a new method to generate family of distributions and called it the family of distributions. This new class of distributions is defined as:

$$
G(x)=\int_{0}^{-\log (1-F(x))} r(t) d t
$$

where $r(t)$ is the probability density function (p. d. f.) of non-negative continuous random variable

The corresponding p. d. f. is given by

$$
g(x)=\frac{f(x)}{1-F(x)} r\{-\log (1-F(x))\} .
$$


Following Alzaatreh et. al., [20], who defined above family, Alzaatreh et. al., [19] defined the Weibull-Pareto distribution by taking $r(t)$ to be the Weibull distribution and $f(x)$ to be the Pareto distribution and Alzaatreh et. al., [21] introduced the gamma-normal distribution by taking $r(t)$ to be the gamma distribution and $f(x)$ to be the normal distribution. Alzaghal et. al., [22] defined a new family of distributions called exponentiated $T-X$ distribution by define the upper limit in Equation (1) to be $-\log \left(1-F^{c}(x)\right)$.

Definition: (See Alzaatreh et al. [19]) :

Let $r(t)$ be the $\mathrm{p}$. d. f. of the random variable $T \in[a, b]$, for $-\infty<a<b<\infty$ and let $W[F(x)]$ be a function of the cumulative distribution function (c. d. f.) of the random variable $X$ such that $W[F(x)]$ satisfies the following conditions:

(i) $W[F(x)] \in[a, b]$,

(ii) $W[F(x)]$ is differentiable and monotonically non-decreasing, and

(iii) $W[F(x)] \rightarrow a$ as $X \rightarrow-\infty$ and $W[F(x)] \rightarrow b$ as $X \rightarrow \infty$

Then according to the above definition, Alzaatreh et. al., [19] defined $T-X$ family of distributions by

$$
G(x)=\int_{a}^{W[F(x)]} r(t) d t
$$

The p. d. f. corresponding to (3) is given by

$$
g(x)=\left\{\frac{d}{d x} W[F(x)]\right\} r\{W[F(x)]\} .
$$

Let $r(t)$ be the probability density function (p. d. f.) on a nonnegative continuous random variable $t$ defined on $[0, \infty)$, and let $F(x)$ denote the c. d. f. of random variable $X$. The c. d. f. of Exponentiated $T-X$ class of distributions for a random variable $x$ is defined by, see Alzaghal et. al., [22]

$$
\begin{aligned}
& G(x)=\int_{0}^{-\log \left(1-F^{c}(x)\right)} r(t) d t \\
= & R\left\{-\log \left(1-F^{c}(x)\right)\right\},
\end{aligned}
$$

and the corresponding p.d. f. is given by

$$
g(x)=\frac{c f(x) F^{c-1}(x)}{1-F^{c}(x)} r\left\{-\log \left(1-F^{c}(x)\right) .\right.
$$

In this new class, the distribution of random variable $t$ is the generator. The new family of distributions generated from equation (3) is called "Exponentiated distributions".We will defined the exponentiated exponentiated exponential-X (EEE-X) distribution from equation (3) by taking to be EE distribution with p. d. f. defined as

$$
r(t)=\alpha \lambda\left(1-e^{-\lambda t}\right)^{\alpha-1} e^{-\lambda t}
$$

Then the p. d. f. of the EEE -X family is given by :

$$
\left.g(x)=c \alpha \lambda(x) F^{c-1}(x)\left(1-F^{c}(x)\right)^{\lambda}\right)^{\alpha-1}\left(1-F^{c}(x)\right)^{\lambda-1}, x>0
$$

The rest of the article is organized as follows. We introduce the EEE-W distribution in Section 2. A range of mathematical properties are considered in Sections 3., quan-tile function, random number generating, skewness, Kurtosis, moment generating function and moments. Two popular entropies are investigated in section 4, namely Shannon entropy and Rényi entropy and we get some numerical values for each one. Estimation by the method of maximum likelihood is presented in Section 5. Finally, Application of the distribution to a real data set is provided in Section 6. 


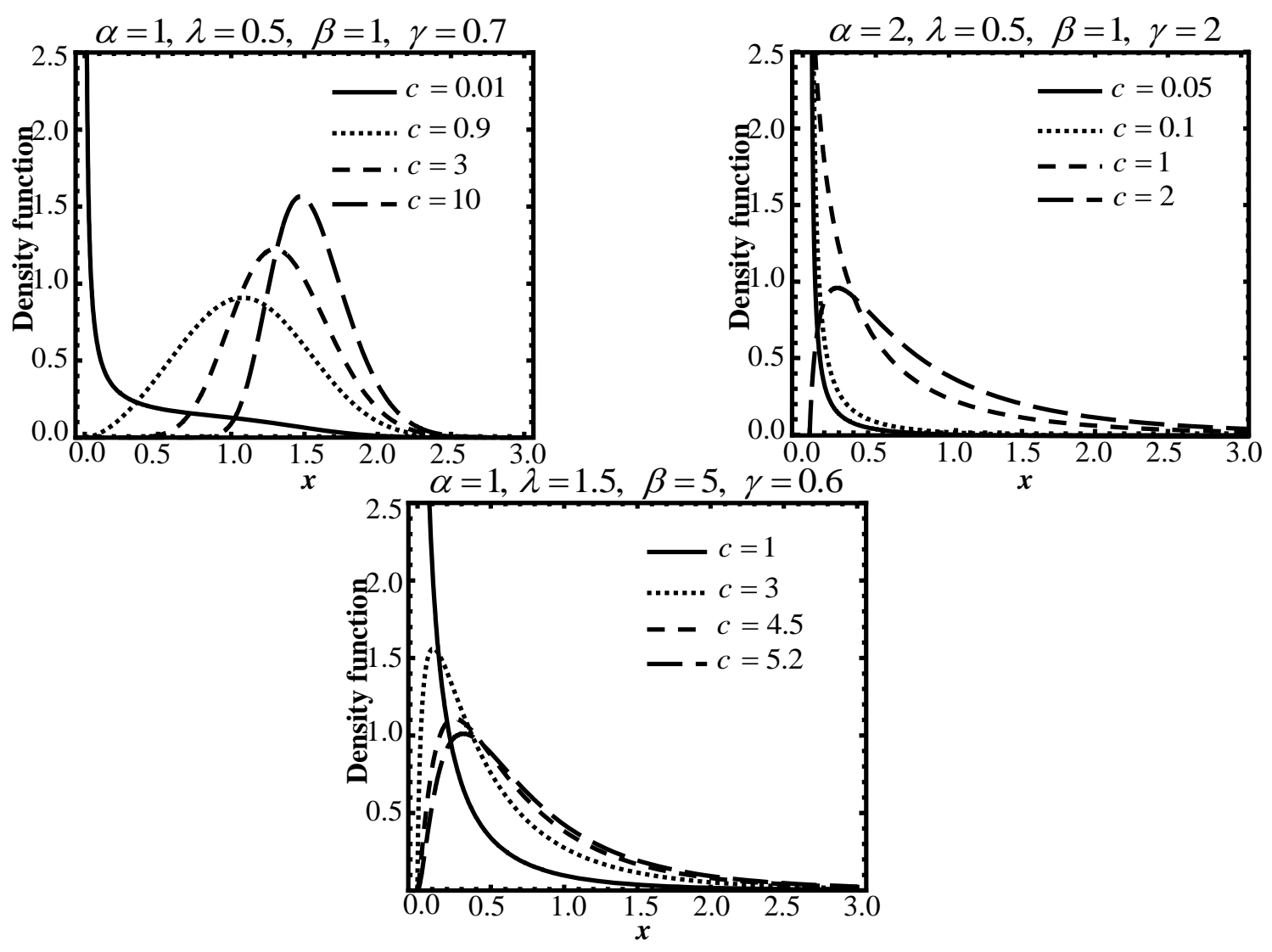

Figure 1. Plots of EEE-W density function for some values of the parameters

\section{THE EEE-WEIBULL DISTRIBUTION:}

If $\mathrm{X} \sim$ Weibull distribution with p. d. f. . $f(x)=\gamma \beta^{\gamma} x^{\gamma-1} e^{-(\beta x)^{\gamma}}, x>0$. Then, from Equation (8), the p. d. f. of EEE-W distribution defined as

$$
\begin{aligned}
& \quad g(x)=c \alpha \lambda\left(\gamma \beta^{\gamma} x^{\gamma-1} e^{-(\beta x)^{\gamma}}\right)\left(1-e^{-(\beta x)^{\gamma}}\right)^{c-1}\left(1-\left(1-\left(1-e^{-(\beta x)^{\gamma}}\right)^{c}\right)^{\lambda}\right)^{\alpha-1} \\
& \times\left(1-\left(1-e^{-(\beta x)^{\gamma}}\right)^{c}\right)^{\lambda-1}
\end{aligned}
$$

From (5) we obtain the c. d. f. of EEE-W distribution as:

(10)

$$
G(x)=\left(1-e^{-\lambda\left(-\log \left\{1-\left(1-e^{-(\beta x)^{\gamma}}\right)^{c}\right\}\right)}\right)^{\alpha}
$$

From Eqs. (9) and (10) we can define the hazard function of EEE-W distribution as follow:

$$
h_{g}(x)=\frac{g(x)}{1-G(x)}=\frac{c \alpha \lambda\left(\gamma \beta^{\lambda} x^{\gamma-1} e^{-(\beta x)^{\gamma}}\right)\left(1-e^{-(\beta x)^{\gamma}}\right)^{c-1} \omega(x)}{1-\left(1-e^{-\lambda\left(-\log \left\{1-\left(1-e^{-(\beta x)^{\gamma}}\right)\right\}\right)}\right)^{\alpha}}
$$

where,

$$
\omega(x)=\left(1-\left(1-\left(1-e^{-(\beta x)^{\gamma}}\right)^{c}\right)^{\lambda}\right)^{\alpha-1}\left(1-\left(1-e^{-(\beta x)^{\gamma}}\right)^{c}\right)^{\lambda-1}
$$



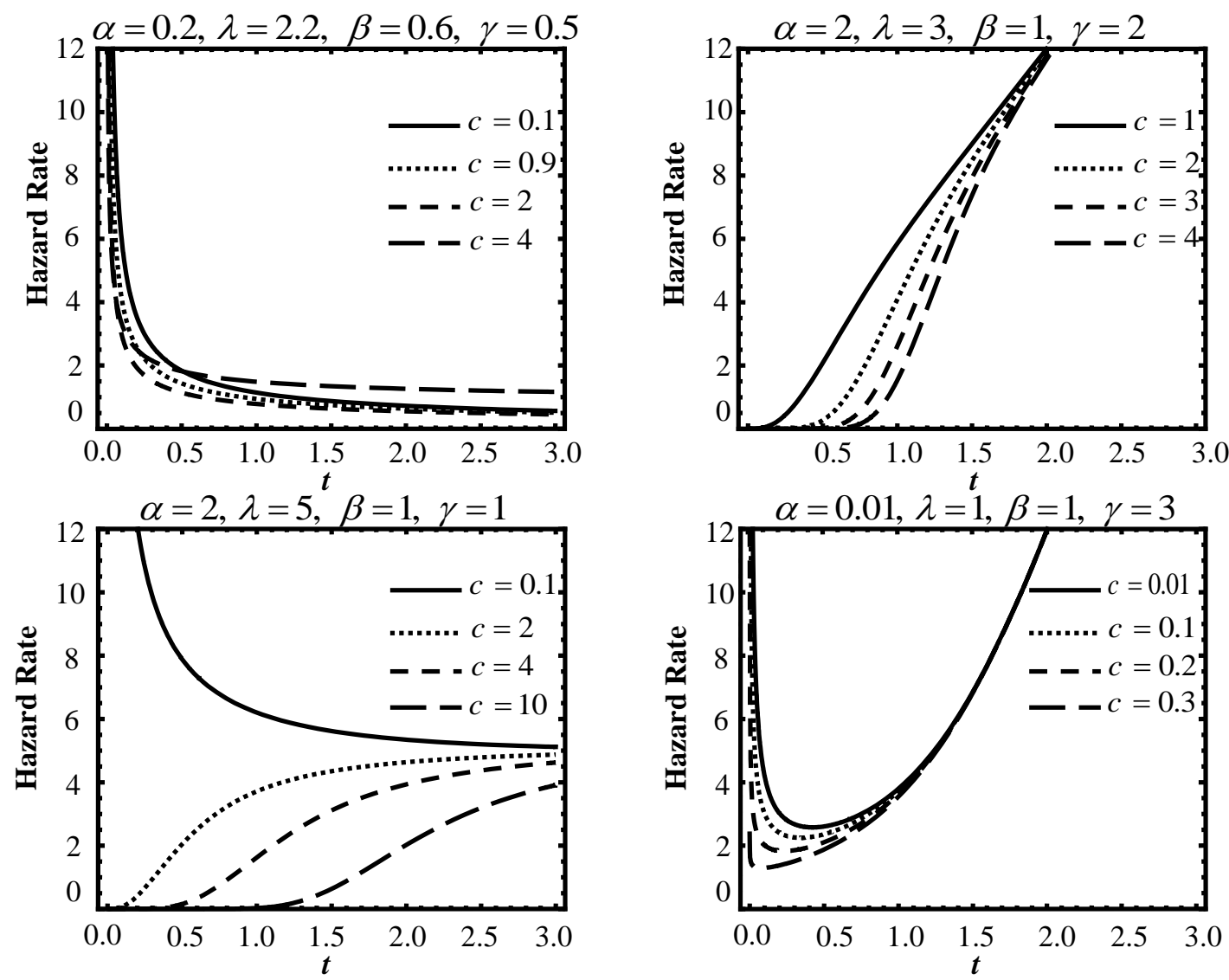

Figure 2. Plots of EEE-W hazard rate function for some specific values of the parameters

Figures 1 and 2 provide some plots of the EEE-W density and hazard rate curves for selected values of parameters. From these figures it is immediate that the p. d. f. ca be reversed $-J$, decreasing, right-skewed and symmetric and the hazard rate function can be decreasing, increasing and bathtub shaped. Hence, the EEE-W distribution can be very useful in fitting different data sets.

\section{STATISTICAL PROPERTIES OF EEE-W.}

In this section, we obtain some statistical properties of the new model, including quantile function, random number generating, Skewness, Kurtosis, moment generating function and moments.

\subsection{Quantile function and random number generating.}

For a non-negative continuous random variable $X$ that follows the EEE-W distribution, the quantile function $x_{q}$ is given by

$$
Q(\lambda)=\frac{\left(-\log \left(1-\left(1-\frac{1-\lambda^{1 / \alpha}}{\lambda}\right)^{1 / c}\right)\right)^{1 / \gamma}}{\beta}
$$

In particular, the distribution median is

$$
Q(0.5)=\frac{\left(-\log \left(1-\left(1-\frac{1-(0.5)^{1 / \alpha}}{0.5}\right)^{1 / c}\right)\right)^{1 / \gamma}}{\beta} .
$$


The random number generation as $\mathrm{X}$ of EEE-W is defined by the following relation

$$
\left(1-e^{-\lambda\left(-\log \left[1-\left(1-e^{-(\beta x)^{\gamma}}\right)^{c}\right]\right)}\right)^{\alpha}=u, \quad \text { where } u \sim U(0,1) .
$$

Thus

$$
x=\frac{\left(-\log \left(1-\left(1-\frac{1-u^{1 / \alpha}}{u}\right)^{1 / c}\right)\right)^{1 / \gamma}}{\beta} .
$$

One can use (13) to generate random numbers when the parameters $\alpha, \beta, \gamma, \lambda$ and $c$ are known

\subsection{Skewness and kurtosis based on quantiles.}

Skewness measures the degree of the long tail and Kurtosis is a measure of the degree of tail heaviness. Based on quantile function $Q(\cdot)$, Galton [23] and Moors [24] defined the Skewness and Kurtosis, respectively, as

$$
S_{G}=\frac{Q(3 / 4)-2 Q(1 / 2)+Q(1 / 4)}{Q(3 / 4)-Q(1 / 4)}
$$

and

$$
K_{M}=\frac{Q(7 / 8)-Q(5 / 8)-Q(3 / 8)+Q(1 / 8)}{Q(6 / 8)-Q(2 / 8)} .
$$

Therefore, Galton's Skewness and Moors' Kurtosis of the quantile function defined by (12) can be get easily. Figure 3 illustrated the graphical representation of the Galton Skewness and Moors Kurtosis as a function of $\gamma$
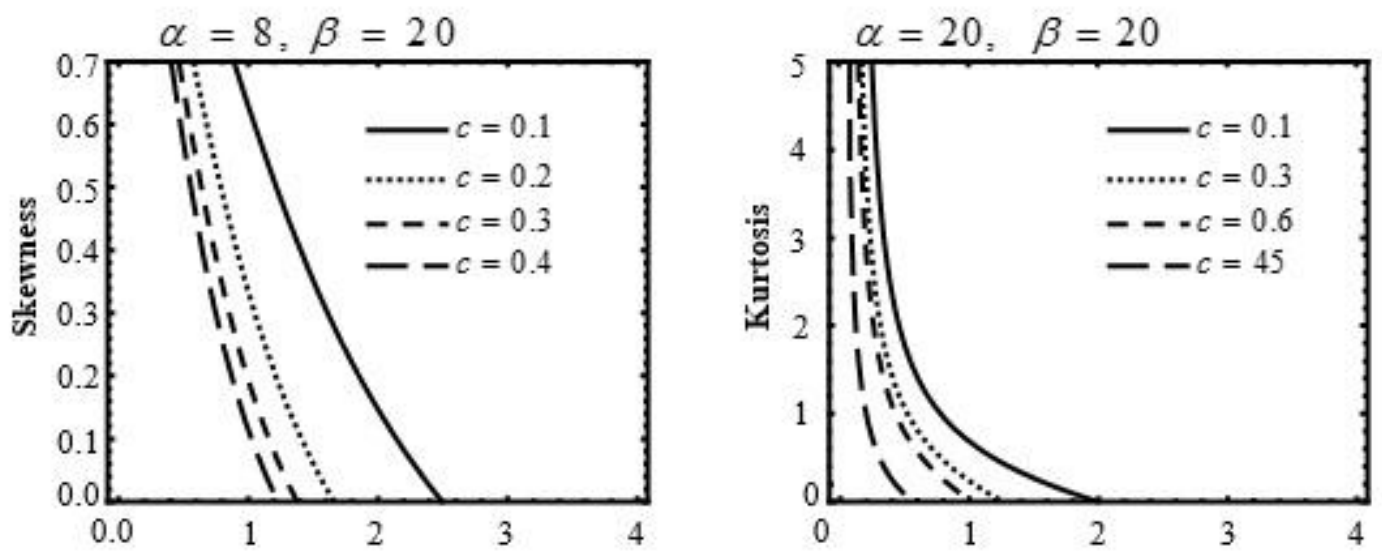

Figure 3. Plots of Galton Skewness and Kurtosis for the EEE-W as a function of $\gamma$.

\subsection{The moment generating function.}

The moment generating function (m.g.f.) of EEE-W distribution can be given as follow

$$
M_{x}(t)=\int_{0}^{\infty} e^{t x} g(x) d x=\int_{0}^{\infty} \sum_{v=0}^{\infty} \frac{t^{v} x^{v}}{v !} g(x) d x
$$

By using the following series expansion 


$$
\left(1-e^{-(\beta x)^{\gamma}}\right)^{c-1}=\sum_{i=0}^{\infty} \frac{(-1)^{i}}{i !}(c-1) e^{-i(\beta x)^{\gamma}}
$$

$$
\begin{aligned}
& \left(1-\left(1-\left(1-e^{-(\beta x)^{\gamma}}\right)^{c}\right)^{\lambda}\right)^{\alpha-1}=\sum_{j=0}^{\infty}\left(\begin{array}{c}
j-\alpha \\
j
\end{array}\right)\left(\begin{array}{c}
-\lambda j+k-1 \\
k
\end{array}\right) \\
& \times \sum_{l=0}^{\infty} \frac{(-1)^{l}}{l !}(c k)^{l} e^{-l(\beta x)^{\gamma}}
\end{aligned}
$$

and

$$
\left(1-\left(1-e^{-(\beta x)^{\gamma}}\right)^{c}\right)^{\lambda-1}=\sum_{m=0}^{\infty}\left(\begin{array}{c}
-\lambda+m \\
m
\end{array}\right) \sum_{n=0}^{\infty} \frac{(-1)^{n}}{n !} e^{-n(\beta x)^{\gamma}}
$$

Hence, the m.g.f. defined as

$$
\begin{array}{r}
M_{x}(t)=1+\sum_{v=1}^{\infty} \frac{t^{v}}{v !} \sum_{i=0}^{\infty} \frac{(-1)^{i}}{i !}(c-1)^{i} \sum_{j=0}^{\infty}\left(\begin{array}{c}
j-\alpha \\
j
\end{array}\right) \sum_{k=0}^{\infty}\left(\begin{array}{c}
-\lambda j+k-1 \\
k
\end{array}\right) \\
\times \sum_{l=0}^{\infty} \frac{(-1)^{l}}{l !}(c k)^{l} \sum_{m=0}^{\infty}\left(\begin{array}{c}
-\lambda+m \\
m
\end{array}\right) \sum_{n=0}^{\infty} \frac{(-1)^{n}}{n !}(c m)^{n} \\
\times\left[\frac{c \alpha \lambda \gamma}{\beta^{v-1}(1+i+l+n)^{\frac{v-1}{\gamma}+1}}\right] \Gamma\left(\frac{v-1}{\gamma}+2\right)
\end{array}
$$

\subsection{The moments.}

The moments of EEE-W distribution can be given as follow

$$
E\left(X^{r}\right)=\int_{0}^{\infty} x^{r} g(x) d x
$$

By using Eqs. (15), (16) and (17), we have

$$
\begin{aligned}
E\left(X^{r}\right)=\sum_{i=0}^{\infty} \frac{(-1)^{i}}{i !}(c-1)^{i} \sum_{j=0}^{\infty}\left(\begin{array}{c}
j-\alpha \\
j
\end{array}\right) \sum_{k=0}^{\infty}\left(\begin{array}{c}
-\lambda j+k-1 \\
k
\end{array}\right) \\
\times \sum_{l=0}^{\infty} \frac{(-1)^{l}}{l !}(c k)^{l} \sum_{m=0}^{\infty}\left(\begin{array}{c}
-\lambda+m \\
m
\end{array}\right) \sum_{n=0}^{\infty} \frac{(-1)^{n}}{n !}(c m)^{n} \\
\times\left[\frac{c \alpha \lambda \gamma}{\beta^{v-1}(1+i+l+n)^{\frac{r-1}{\gamma}+1}}\right] \Gamma\left(\frac{r-1}{\gamma}+2\right)
\end{aligned}
$$




\section{Remark:}

The mean and the variance of EEE-W are reported in Table 1 for some values of $c, \alpha, \lambda, \beta$ and $\gamma$. It is observed that, mean and variance decreasing as the values of the parameters $\lambda, \beta$ and $\gamma$ increasing. When the values of $c$ and $\alpha$ are increase, the mean and variance increase.

Table 1: Mean and variance for some arbitrary parameter values.

\begin{tabular}{|c|c|c|}
\hline Parameters & $\alpha=1, \lambda=1$ & $\beta=1, \gamma=1$ \\
\hline$c$ & Mean & Variance \\
\hline 0.1 & 0.153461 & 0.211635 \\
\hline 0.5 & 1.08677 & 0.710132 \\
\hline 1.5 & 1.28037 & 1.15458 \\
\hline 2 & 1.5 & 1.25 \\
\hline$\alpha$ & $c=1, \lambda=1$ & $\beta=1, \gamma=1$ \\
\hline 0.1 & 0.153461 & 0.211635 \\
\hline 0.5 & 0.613706 & 0.710132 \\
\hline 1.5 & 1.28037 & 1.15458 \\
\hline 1.9 & 1.45972 & 1.23397 \\
\hline$\lambda$ & $c=1, \alpha=1$ & $\beta=1, \gamma=1$ \\
\hline 0.4 & 2.5 & 6.25 \\
\hline 0.6 & 1.66667 & 2.77778 \\
\hline 0.8 & 1.25 & 1.5625 \\
\hline 1.2 & 0.833333 & 0.694444 \\
\hline$\beta$ & $c=1, \alpha=1$ & $\beta=1, \gamma=1$ \\
\hline 0.3 & 3.33333 & 11.1111 \\
\hline 0.6 & 1.66667 & 2.77778 \\
\hline 0.9 & 1.11111 & 1.23457 \\
\hline 1.2 & 0.833333 & 0.694444 \\
\hline$\gamma$ & $c=1, \alpha=1$ & $\beta=1, \gamma=1$ \\
\hline 0.4 & 3.32335 & 108.955 \\
\hline 0.8 & 1.133 & 2.03965 \\
\hline 1.2 & 0.940656 & 0.619742 \\
\hline 1.5 & 0.902745 & 0.37569 \\
\hline
\end{tabular}

\section{ENTROPIES.}

Entropy is measure of the randomness of systems and it is widely used in areas like physics, molecular imaging of tumors and sparse kernel density estimation. Two popular entropy measures are the Shannon entropy (Shannon [25]) and Rényi entropy (Rényi [26])

$$
\eta_{x}=E(-\log g(x)), \quad \text { and } \quad I_{R(\varepsilon)}=\frac{1}{1-\varepsilon} \log \left[\int_{R} f^{\varepsilon}(x) d x\right],
$$


respectively, where $\varepsilon>0$ and $\varepsilon \neq 1$.

\subsection{Shannon entropy.}

The Shannon entropy for the $E T-X$ family defined by Alzaghal et. al., [22] as

$$
\eta_{x}=-\log c-E\left(\log f\left(F^{-1}\left\{1-e^{-t}\right\}^{1 / c}\right)\right)+\frac{1-c}{c} E\left(\log \left\{1-e^{-t}\right\}\right)-\mu_{T}+\eta_{T} .
$$

Then, from Equation (21) the Shannon entropy of EEE-W distribution is defined by

$$
\begin{aligned}
& \eta_{x}=-\log c-E\left(\log f\left(F^{-1}\left\{1-e^{-t}\right\}^{1 / c}\right)\right)+\frac{1-c}{c} E\left(\log \left\{1-e^{-t}\right\}\right) \\
& +\frac{\alpha}{\lambda} \sum_{i=1}^{n}(-1)^{i} c(\alpha-1, i) \frac{1}{(1+i)^{2}}-\log (\alpha \lambda)-\phi(1)+\alpha \phi(\alpha+1),
\end{aligned}
$$

where,

$$
\begin{aligned}
\mu_{T} & =\frac{\alpha}{\lambda} \sum_{i=1}^{n}(-1)^{i} c(\alpha-1, i) \frac{1}{(1+i)^{2}}, \\
c(\alpha-1, i) & =\frac{(\alpha-1)(\alpha-2) \ldots(\alpha-i)}{i !}
\end{aligned}
$$

and

$$
\eta_{T}=-\log (\alpha \lambda)-\phi(1)+(1-\alpha)+\alpha \phi(\alpha+1),
$$

are the mean and Shannon entropy for the Weibull distribution, respectively.

\section{Remark.}

Some numerical values for the Shannon entropy are displayed at Table 2. It can be observed that this entropy decreasing with increasing $\lambda, \beta$ and $\gamma$ and can have negative values.

Table 2: Shannon entropy for some arbitrary parameter values.

\begin{tabular}{|c|c|c|}
\hline Parameters & $\alpha=1, \lambda=1$ & $\beta=2, \gamma=1$ \\
\hline$C$ & & Shannon entropy \\
\hline 2 & & 0.613706 \\
\hline 2.5 & & 0.670934 \\
\hline 3.5 & & 0.734462 \\
\hline 5 & & 0.780748 \\
\hline$\alpha$ & $c=1, \lambda=3$ & $\beta=0.4, \gamma=1$ \\
\hline 1.5 & & 0.815923 \\
\hline 2 & & 0.833236 \\
\hline 3 & & 0.850365 \\
\hline 5 & & 0.863935 \\
\hline$\lambda$ & $c=2, \alpha=1$ & $\beta=2, \gamma=1$ \\
\hline 2 & & 0.0872251 \\
\hline 3 & & -0.201573 \\
\hline 4 & & -0.398779 \\
\hline 5 & & -0.547716 \\
\hline$\beta$ & $c=2, \alpha=3$ & $\lambda=1, \gamma=1$ \\
\hline 3 & & 0.392962 \\
\hline 4 & & 0.10528 \\
\hline 5 & & -0.117864 \\
\hline
\end{tabular}




\begin{tabular}{|c|c|c|}
\hline 7 & & -0.454336 \\
\hline$\gamma$ & $c=1, \alpha=2$ & $\lambda=1, \beta=3$ \\
\hline 2 & & -0.542872 \\
\hline 3 & & -0.967659 \\
\hline 4 & & -1.265 \\
\hline 5 & & -1.49394 \\
\hline
\end{tabular}

\subsection{Rényi entropy.}

Let $X \sim$ EEE-W $(c, \alpha, \lambda, \beta, \gamma)$, then the corresponding Rényi entropy is obtained as:

$$
\begin{gathered}
I_{R(\varepsilon)}=\frac{1}{1-\varepsilon} \log \left(\int _ { 0 } ^ { \infty } \left(c \alpha \lambda ( \gamma \beta ^ { \gamma } x ^ { - \gamma - 1 } e ^ { - ( \beta x ) ^ { \gamma } } ) \left(1-\left(1-\left(1-e^{-(\beta x)^{\gamma}}\right)^{c}\right)^{\gamma)^{\alpha-1}}\right.\right.\right. \\
\left.\quad \times\left(1-\left(1-e^{-(\beta x)^{\gamma}}\right)^{c}\right)^{\gamma} d x\right)
\end{gathered}
$$

\section{Remark.}

Some numerical values for the Renyi entropy are displayed at Table 3. It can be observed that this entropy decreasing with increasing $\lambda$ and $\beta$ and can have negative values.

Table 3: Rényi entropy for some arbitrary parameter values.

\begin{tabular}{|c|c|c|}
\hline Parameters & $\alpha=0.2, \lambda=3$ & $\beta=0.4, \gamma=1$ \\
\hline$C$ & & Rényi entropy \\
\hline 1 & & 0.249183 \\
\hline 1.1 & & 0.374287 \\
\hline 1.2 & & 0.484313 \\
\hline 1.3 & & 0.582012 \\
\hline$\alpha$ & $c=1, \lambda=3$ & $\beta=0.4 \quad \gamma=1$ \\
\hline 0.01 & & -2.51181 \\
\hline 0.02 & & -1.83229 \\
\hline 0.03 & & -1.44028 \\
\hline 0.05 & & $\beta=0.4 \quad \gamma=1$ \\
\hline$\lambda$ & $c=1, \alpha=0.05$ & 0.0473772 \\
\hline 1.1 & & -0.0396342 \\
\hline 1.2 & & -0.1937850 \\
\hline 1.4 & & -0.3273160 \\
\hline 1.6 & & $\lambda=1.8 \quad \gamma=1$ \\
\hline$\beta$ & $c=1, \alpha=0.05$ & 0.248048 \\
\hline 0.2 & & -0.445099 \\
\hline 0.4 & & -0.850564 \\
\hline 0.6 & & -1.13825 \\
\hline 0.8 & & \\
\hline & & \\
\hline
\end{tabular}




\begin{tabular}{|c|c|c|}
\hline$\gamma$ & $c=1, \alpha=0.05$ & $\lambda=1.8 \quad \beta=0.8$ \\
\hline 1.1 & & -1.0999 \\
\hline 1.3 & & -1.01836 \\
\hline 1.5 & & -0.937665 \\
\hline 1.7 & & -0.861333 \\
\hline
\end{tabular}

\section{MAXIMUM LIKELIHOOD ESTIMATION.}

Consider the random variable $X$ follows Weibull distribution and let $\theta=(c, \alpha, \lambda, \beta, \gamma)$ be the parameter Vector. The $\log$-likelihood $\ell=\ell(\theta)$ is given by

$$
\begin{aligned}
\ell= & n \log c+n \log \alpha+n \log \lambda+n \log \gamma+n \log \beta^{\gamma}+(\gamma-1) \sum_{i=1}^{n} \log x_{i}-\beta^{\gamma} \sum_{i=1}^{n} x_{i}^{\gamma} \\
& +(c-1) \sum_{i=1}^{n} \log \left(1-e^{-\left(\beta x_{i}\right)^{\gamma}}\right)+(\alpha-1) \sum_{i=1}^{n} \log \left(1-\left(1-\left(1-e^{-\left(\beta x_{i}\right)^{\gamma}}\right)^{c}\right)^{\lambda}\right) \\
& +(l-1) \sum_{i=1}^{n} \log \left(1-\left(1-e^{-\left(\beta x_{i}\right)^{\gamma}}\right)^{c}\right)
\end{aligned}
$$

On taking the partial derivatives of the log-likelihood in Equation (24) with respect to $c, \alpha, \lambda, \beta$ and $\gamma$ and equating the derivatives to zero, we have

$$
\begin{aligned}
\frac{\partial \ell}{\partial c}=\frac{n}{c}+ & \sum_{i=1}^{n} \log \left(1-e^{-\left(\beta x_{i}\right)^{\gamma}}\right) \\
& +(\alpha-1) \sum_{i=1}^{n} \frac{\lambda\left(1-\left(1-e^{-\left(\beta x_{i}\right)^{\gamma}}\right)^{c}\right)^{\lambda-1}\left(1-e^{-\left(\beta x_{i}\right)^{\gamma}}\right)^{c} \log \left(1-e^{-\left(\beta x_{i}\right)^{\gamma}}\right)}{1-\left(1-\left(1-e^{-\left(\beta x_{i}\right)^{\gamma}}\right)^{c}\right)^{\lambda}} \\
+(\lambda-1) \sum_{i=1}^{n} \frac{-\left(1-e^{-\left(\beta x_{i}\right)^{\gamma}}\right)^{c} \log \left(1-e^{-\left(\beta x_{i}\right)^{\gamma}}\right)}{1-\left(1-e^{-\left(\beta x_{i}\right)^{\gamma}}\right)^{c}}=0 & \\
\frac{\partial \ell}{\partial \alpha}= & \frac{n}{\alpha}+\sum_{i=1}^{n} \log \left(1-\left(1-\left(1-e^{-\left(\beta x_{i}\right)^{\gamma}}\right)^{c}\right)^{\lambda}\right)=0, \\
\frac{\partial \ell}{\partial \lambda}= & \frac{n}{\lambda}+(\alpha-1) \sum_{i=1}^{n} \frac{-\left(\left(1-\left(1-e^{-\left(\beta x_{i}\right)^{\gamma}}\right)^{c}\right)^{\lambda}\right) \log \left(1-\left(1-e^{-\left(\beta x_{i}\right)^{\gamma}}\right)^{c}\right)}{\left.1-\left(1-e^{-\left(\beta x_{i}\right)^{\gamma}}\right)^{c}\right)^{\lambda}} \\
& +\sum_{i=1}^{n} \log \left(1-\left(1-e^{\left.\left.-\left(\beta x_{i}\right)^{\gamma}\right)^{c}\right)=0,}\right.\right. \\
\frac{\partial \ell}{\partial \gamma}= & \frac{n}{\gamma}+n \log \beta+\sum_{i=1}^{n} \log x_{i}-\beta^{\gamma} \sum_{i=1}^{n} x_{i}^{\gamma} \log x_{i}-\beta^{\gamma} \log \beta \sum_{i=1}^{n} x_{i}^{\gamma} \\
& +(c-1) \sum_{i=1}^{n} \frac{\left(\beta x_{i}\right)^{\gamma} \log \left(\beta x_{i}\right) e^{-\left(\beta x_{i}\right)^{\gamma}}}{1-e^{-\left(\beta x_{i}\right)^{\gamma}}}
\end{aligned}
$$




$$
\begin{gathered}
+(\lambda-1) \sum_{i=1}^{n} \frac{-c\left(1-e^{-\left(\beta x_{i}\right)^{\gamma}}\right)^{c-1}\left(\beta x_{i}\right)^{\gamma} \log \left(\beta x_{i}\right) e^{-\left(\beta x_{i}\right)^{\gamma}}}{1-\left(1-e^{-\left(\beta x_{i}\right)^{\gamma}}\right)^{c}}=0, \\
\frac{\partial \ell}{\partial \beta}=\frac{n \gamma}{\beta}-\gamma \beta^{\gamma-1} \sum_{i=1}^{n} x_{i}^{\gamma}+(c-1) \sum_{i=1}^{\infty} \frac{\gamma \beta^{\gamma-1} x_{i}^{\gamma} e^{-\left(\beta x_{i}\right)^{\gamma}}}{1-e^{-\left(\beta x_{i}\right)^{\gamma}}}+(\alpha-1) \times \\
\sum_{i=1}^{n} \frac{\lambda\left(1-\left(1-e^{-\left(\beta x_{i}\right)^{\gamma}}\right)^{c}\right)^{\lambda-1} c\left(1-e^{-\left(\beta x_{i}\right)^{\gamma}}\right)^{c-1} \gamma \beta^{\gamma} x_{i}^{\gamma-1} x_{i}^{\gamma-1} e^{-\left(\beta x_{i}\right)^{\gamma}}}{1-\left(1-\left(1-e^{-\left(\beta x_{i}\right)^{\gamma}}\right)^{c}\right)^{\lambda}} \\
=0 .
\end{gathered}
$$

Solving these equations numerically using the statistical software Mathimatica package yields the maximum likelihood estimators (MLEs) $\hat{\theta}=(\hat{\alpha}, \hat{\beta}, \hat{\lambda}, \hat{\gamma}, \hat{c})$ of $\theta=(\alpha, \beta, \lambda, \gamma, c)$

\section{REAL DATA APPLICATION.}

Here, we illustrate the applicability of EEE-W distribution by considering the

following dataset. We fitted the following distributions to data set: Weibull distribution, Gamma distribution, modified Weibull distribution and transmuted Weibull distribution:

- Weibull distribution $(\mathrm{W}(\beta, \gamma))$ :

$$
g(x)=\gamma \beta^{\gamma} x^{\gamma-1} e^{-(\beta x)^{\gamma}}, \quad x, \gamma, \beta>0,
$$

- Gamma distribution $(\mathrm{Ga}(k, \theta))$ :

$$
g(x)=\frac{1}{\Gamma[k] \theta^{k}} x^{k-1} e^{-\frac{x}{\theta}}, \quad x, k, \theta>0,
$$

- Modified Weibull (MW $(\delta, \beta, \gamma))$ :

$$
g(x)=\left(\delta+\beta \gamma x^{\gamma-1}\right) e^{-x^{\gamma} \beta-x \delta}, \quad x, \delta, \beta, \gamma>0,
$$

- Transmuted Weibull (TW $(\eta, \sigma, \lambda))$ :

$$
g(x)=\frac{\eta}{\sigma}\left(\frac{x}{\sigma}\right)^{\eta-1} e^{-\left(\frac{x}{\sigma}\right)^{\eta}}\left(1-\lambda+2 \lambda e^{-\left(\frac{x}{\sigma}\right)^{\eta}}\right), \quad x, \eta, \sigma>0,|\lambda|>1 .
$$

For illustrative purposes, we estimate the unknown parameters of each distribution by the maximum-likelihood method, and with these obtained estimates; we obtain the values of Akaike Information Criterion (AIC), and Hannan-Quinn Information Criterion (HQIC). The following dataset acts the breaking stress of carbon fibers of $50 \mathrm{~mm}$ in length (Nichols and Padgett [27]) and listed in Table 4.

Additionally, to compare the models, we used four other criterions:

- Kolmogorov-Smirnov test statistics (K-S) - small value is good.

- The P-value from the chi-square goodness-of-fit test-large value is good.

- Negative log-likelihood-small value is good.

- Cramer-von Mises $\left(W^{*}\right)$ goodness-of-fit statistic-small value is good.

- Anderson-Darling $\left(A^{*}\right)$ goodness-of-fit statistic-small value is good.

The results for the dataset are presented in Tables 5, 6, 7 and 8. From these results indicate that the EEE-W distribution has the smallest values of the AIC, HQIC, K-S, , and the largest value of $p$-value are obtained for the EEE-W 
distribution. Therefore, we conclude that the EEE-W distribution provides the best fit among the compared distributions. Table 9 gives some descriptive statistics for dataset and it is noted that the dataset has negative Kurtosis.

Table 4: Breaking stress of carbon fibers data.

\begin{tabular}{|l|l|l|l|l|l|l|l|l|l|l|}
\hline 3.70 & 2.12 & 2.95 & 4.70 & 1.25 & 3.22 & 1.69 & 3.27 & 2.87 & 1.47 & 3.11 \\
\hline 3.65 & 2.74 & 3.15 & 2.97 & 2.03 & 4.38 & 3.39 & 3.28 & 3.09 & 1.87 & 3.15 \\
\hline 4.90 & 4.42 & 2.73 & 1.08 & 3.39 & 1.89 & 1.84 & 2.81 & 4.20 & 3.33 & 2.55 \\
\hline 3.31 & 1.57 & 2.41 & 2.50 & 2.56 & 2.96 & 2.88 & 0.39 & 3.68 & 2.48 & 0.85 \\
\hline 1.61 & 3.31 & 2.67 & 3.19 & 3.60 & 1.80 & 2.35 & 2.82 & 2.05 & 3.65 & 3.75 \\
\hline 2.43 & 2.79 & 2.85 & 2.93 & 3.22 & 3.11 & 2.53 & 2.55 & 2.59 & 2.03 & 1.61 \\
\hline
\end{tabular}

Table 5: The MLEs of the parameters for some models fitted to the Breaking stress of carbon fibers data.

\begin{tabular}{|l|c|c|c|c|c|}
\hline \multicolumn{1}{|c|}{ Distribution } & \multicolumn{5}{|c|}{ Estimates } \\
\hline$W(\beta, \gamma)$ & 0.345 & 2.000 & - & - & - \\
\hline$G a(k, \theta)$ & 13.384 & 0.200 & - & - & - \\
\hline$M W(\delta, \beta, \gamma)$ & $1.1 \times 10^{-25}$ & 0.063 & 2.543 & - & - \\
\hline$T W(\eta, \sigma, \lambda)$ & 1.899 & 2.355 & 0.983 & - & - \\
\hline$E E E W(C, \alpha, \lambda, \beta, \gamma)$ & $\mathbf{0 . 9 8 6}$ & $\mathbf{0 . 8 1 9}$ & $\mathbf{1 . 6 3 0}$ & $\mathbf{0 . 2 7 3}$ & $\mathbf{3 . 8 8 4}$ \\
\hline
\end{tabular}

Table 6: The values of AIC and HQIC statistics for some models fitted to Breaking stress of carbon fibers data.

\begin{tabular}{|l|c|c|}
\hline \multicolumn{1}{|c|}{ Distribution } & AIC & HQIC \\
\hline$W(\beta, \gamma)$ & 200.417 & 202.147 \\
\hline$E W(\alpha, \gamma, \beta)$ & 203.213 & 205.809 \\
\hline$G a(k, \theta)$ & 201.308 & 203.039 \\
\hline$M W(\delta, \beta, \gamma)$ & 187.502 & 190.098 \\
\hline$T W(\eta, \sigma, \lambda)$ & 185.752 & 188.348 \\
\hline$E E E W(C, \alpha, \lambda, \beta, \gamma)$ & $\mathbf{1 8 1 . 8 4 9}$ & $\mathbf{1 8 6 . 2 2}$ \\
\hline
\end{tabular}

Table 7: The values of K-S, p-value and -Log L statistics for some models fitted to Breaking stress of carbon fibers data.

\begin{tabular}{|l|c|c|c|}
\hline \multicolumn{1}{|c|}{ Distribution } & K-S & p-value & $-\log \mathbf{L}$ \\
\hline W & 0.226512 & 0.00228941 & 98.2083 \\
\hline Ga & 0.141662 & 0.14139700 & 98.6540 \\
\hline MW & 0.177923 & 0.03063720 & 90.7509 \\
\hline TW & 0.151172 & 0.09792410 & 89.8759 \\
\hline EEEW & $\mathbf{0 . 0 8 1 0 5 9 8}$ & $\mathbf{0 . 7 7 8 6 7 9 0 0}$ & $\mathbf{8 5 . 9 4 6 8}$ \\
\hline
\end{tabular}


Table 8: The values of $W^{*}$ and $A^{*}$ statistics for some models fitted to Breaking stress of carbon fibers data.

\begin{tabular}{|c|c|c|}
\hline Distribution & $W^{*}$ & $A^{*}$ \\
\hline W & 1.018880 & 5.20603 \\
\hline Ga & 0.402684 & 2.63673 \\
\hline MW & 0.516374 & 2.62049 \\
\hline TW & 0.336408 & 1.73131 \\
\hline EEEW & $\mathbf{0 . 2 6 7 0 2}$ & $\mathbf{0 . 4 9 3 6 3}$ \\
\hline
\end{tabular}

Table 9: Descriptive statistics of the EEE-W distribution for the Breaking stress of carbon fibers data

\begin{tabular}{|c|c|c|c|c|}
\hline Mean & Median & SD & MD-mean & MD-median \\
\hline 2.75955 & 2.835 & 0.891455 & 0.683223 & 0.678939 \\
\hline Skewness & Kurtosis & S. Entropy & Min. & Max. \\
\hline-0.13046 & 0.17421 & 0.891455 & 1.25 & 4.90 \\
\hline
\end{tabular}

$\mathrm{MD}=$ Mean deviation, $\mathrm{S}=$ Shannon

\section{REFERENCES}

1. Jhonson, N. L., Kotz, S., and Balakrishnan, N. (1994). Continuous univariate distributions. I. Wiley, Newyork.

2. Jhonson, N. L., Kotz, S., and Balakrishnan, N. (1995). Continuous univariate distributions. II. Wiley, Newyork.

3. Hansen, B. E., (1994). Auto regressive conditional density estimation. Intern-ational Economic Review 35, 705-730.

4. Azzalini, A., and Capitanio, A. (2003). Distributions generated by perturbation of summetry with emphasis an a multivariate skew t-distribution. Journal of the Royal Statistical Society. Series B 65, 397-389.

5. Eugene, N., Lee, C., and Famoye, F. (2002). Beta-normal distribution and its applications. Communication in Statistics-Theory and Methods 31, 497-512.

6. Aas, K., and Haff, I. H. (2006). The generalized hyperbolic skew student's t-distribution. Journal of Financial Econometrics 4, 275-309.

7. Gurvich, M. R., Dibenedetto, A. T. and Ranade, S. V. (1997). A new statistical distribution for characterizing the random strength of brittle materials. Journal of Materials Science 32, 2559-2564.

8. Bebbington, M. S., Lai, C. D. and Zitikis, R. (2007). A flexible Weibull extension. Reliability Engineering and System Safety 92(6), 719-726.

9. Zhang, T. and Xie, M. (2011). On the upper truncated Weibull distribution and its reliability implications. Reliability Engineering and System Safety 96(1), 194-200.

10. Xie, M., Tang, Y. and Goh, T. N. (1995). A modified Weibull extension with bathtub-shaped failure rate function. Reliability Engineering and System Safety 52, 87-93.

11. Carrasco, M., Ortega, E. M. and Cordeiro, G. M. (2008). A generalized modified Weibull distribution for lifetime modeling. Computational Statistics and Data Analysis 53(2), 450-462.

12. Sarhan, A. M. and Zaindin, M. (2009). Modified Weibull distribution. Applied Sciences 11, 123-136.

13. Almalki, S. J. and Yuan, J. (2013). A new modified Weibull distribution. Reliability Engineering and System Safety $111,164-170$.

14. Xie, M. and Lai, C. D. (1995). Reliability analysis using an additive Weibull model with bathtub-shaped failure rate function. Reliability Engineering and System Safety 52, 87-93.

15. Cordeiro, G. M., and de Castro, M. (2011). A new family of generalized distributions. Journal of Statistical.81(7),883898.

16. Nadarajah, S. and Kotz, S. (2004). The beta Gumbel distribution. Mathematical Problems in Engineering 10, $323-$ 332. 
17. Nadarajah, S. and Gupta, A. K. (2004). The beta Fréchet distribution. Far East Journal of Theoretical Statistics 15, 15-24.

18. Nadarajah, S. and Kotz, S. (2005). The beta exponential distribution. Reliability Engineering and System Safety 91 , 689-697.

19. Alzaatreh, A., Lee, C., and Famoye, F. (2013). A new method for generating families of continuous distributions. METRON 71(1), 63-79.

20. Alzaatreh, A., Famoye, F., and Lee, C. (2013). Weibull-Pareto distribution and its applications. Communications in Statistics-Theory and Methods 42(9), 1673-1691.

21. Alzaatreh, A., Famoye, F., and Lee, C. (2014). The gamma-normal distribution: properties and applications. Computational Statistics and Data Analysis 69, 67-80.

22. Alzaghal, A., Famoye, F., and Lee, C. (2013). Exponentiated T-X family of distributions with some applications. International Journal of Statistics and Probability 2(3), 31-49.

23. Galton, F. (1883) Enquiries on to Human Faculty and its Developments. Macmillan \& Company. London.

24. Moors, J. J. (1998). A quantile alternative for kurtosis. Journal of The Royal Statistical Society. Series $D($ the statistician) $37,25-32$.

25. Shannon, C. E. (1948). A Mathematical Theory of Communication .Bell System Technical Journal 27, 379-432.

26. Rényi, A. (1961). On measures of entropy and information. In Proceedings of the 4th Berkeley symposium on Mathematical Statistics and Probability I, 547-561. Berkeley: Univ. California Press. MR0132570.

27. [27] Nichols, M. D. and Padgett, W. J. (2006). A boot strap Control for Weibull Percentiles. Quality and Reliability Engineering International 22, 141-151.

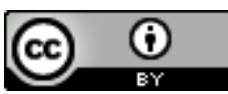

This work is licensed under a Creative Commons Attribution 4.0 International License. 